\title{
Crystal structure of an As(III) S-adenosylmethionine methyltransferase with both bound ligand and product demonstrates a conformational change in the $\mathrm{N}$ - terminal domain during catalysis
}

\author{
${ }^{1}$ Charles Packianathan, ${ }^{2}$ Palani Kandavelu, Banumathi Sankaran ${ }^{3}$ and ${ }^{1}$ Barry P. Rosen
}

${ }^{1}$ Department of Cellular Biology and Pharmacology, Herbert Wertheim College of Medicine, Florida International University, Miami, Florida 33199, U.S.A.

${ }^{2}$ SER-CAT and the Department of Biochemistry and Molecular Biology, University of Georgia, Athens, GA 30602, U.S.A.

${ }^{3}$ Berkeley Center for Structural Biology, Lawrence Berkeley Laboratory, 1 Cyclotron Road, Bldg 6R2100, Berkeley, CA 94720.

Arsenic is the most pervasive environmental toxic substance. Arsenic is ubiquitous in water, soil and food and ranks first on the Environmental Protection Agency's Superfund Priority List of Hazardous Substances. Arsenic(III) S-adenosylmethionine (SAM) methyltransferase (AS3MT in animals and ArsM in microbes) is the key enzyme of arsenic biotransformation, catalyzing the methylation of inorganic arsenite to methyl, dimethyl and trimethyl products. As(III) methyltransferases are found in members of every kingdom, from bacteria to humans (EC 2.1.1.137). In the human liver, hAS3MT converts inorganic arsenic into more the toxic and carcinogenic methylated species. CmArsM, an ortholog of hAS3MT from the thermophilic eukaryotic alga Cyanidioschyzon merolae, was purified and expressed it in Escherichia coli. Here we report a crystal structure of CmArsM with arsenic (III) bound in the As binding site and Sadenosyl L- homocysteine (SAH) in the SAM binding site at $2.84 \AA$ resolution . The crystals belong to the tetragonal space group $P 4_{2} 22$, with unit cell parameters $a=101.80 \AA, b=101.80 \AA, c=175.01$ $\AA, \alpha=\beta=\gamma=90.0^{\circ}$, and two molecules in the asymmetric unit. As(III) is bound to three conserved cysteine residues, Cys44, Cys174 and Cys224. A conformational change in the N-terminal domain of $\mathrm{CmArsM}$ with bound $\mathrm{As}$ (III) and SAH alters the secondary structural elements, which results in movement of a loop containing conserved cysteine residue Cys72 toward the arsenic binding site. We previously reported the structure of CmArsM with bound SAM (PDB ID: 4FR0), which has a disulfide bond between Cys74 and Cys174. The As(III)- and SAH-bound structure lacks this disulfide bong. From these results we propose a new model for the reaction pathway involving a disulfide bond cascade during catalysis. Crystallization trials of CmArsM with bound ligands are in progress that have the potential to elucidate details of the disulfide cascade. Supported by National Institutes of Health grants R01 GM55425 and R01 ES023779 to B.P.R. and HWCOM pilot project funding to C.P. We acknowledge the synchrotron beam lines at APS, Chicago and ALS, Berkeley for data collection.

1) Marapakala, K. et al., (2015). Acta Cryst. D71, 505-515.

2) Packianathan, C. et al., (2014). Acta Cryst. F70, 1385-1388.

3) Ajees, A. A . et al., (2012). Biochemistry. 51, 5476-5485.

4) Jiaojiao Li, Packianathan C, Toby G. Rossman and Barry P. Rosen. Chemical Res Toxicology (2017), 30(7), 1481-1491. 